\title{
MONITORIZAÇÃO AMBULATORIAL DA PRESSÃO ARTERIAL (MAPA)
}

\author{
ARTERIAL BLOOD PRESSURE MONITORING (ABPM)
}

\section{Fernando Nobre}

\begin{abstract}
Coordenador da Unidade de Hipertensão do Hospital das Clínicas da Faculdade de Medicina de Ribeirão Preto da Universidade de São Paulo

CorResPondÊnCIA: Hospital das Clínicas da Faculdade de Medicina de Ribeirão Preto da Universidade de São Paulo, Seção de Cardiologia, Unidade de Hipertensão - Campus Universitário - CEP: 14048-900 - Ribeirão Preto - SP
\end{abstract}

NOBRE F. Monitorização ambulatorial da pressão arterial (MAPA). Medicina, Ribeirão Preto, 29: 250-257, abr./set. 1996

RESUMO: Monitorização Ambulatorial da Pressão Arterial (MAPA) é uma técnica que permite a observação das variações tensionais durante 24 ou mais horas, através de medidas programadas e indiretas da pressão arterial. Vantagens, limitações, indicações e utilidades sobre esta metodologia são discutidas nesta revisão.

UNITERMOS: Hipertensão. Monitorização Ambulatorial da Pressão Arterial.

\section{INTRODUÇÃO}

Monitorização Ambulatorial da Pressão Arterial (MAPA) é uma técnica através da qual medidas múltiplas e indiretas da pressão arterial podem ser obtidas durante 24 ou mais horas consecutivas, com um mínimo de desconforto, durante as atividades diárias do paciente ${ }^{1}$.

A possibilidade de se obter um curva representativa das variações pressóricas, em determinado período, faz com que se possa ter uma visão dinâmica do comportamento tensional e não apenas uma observação, meramente, estática, refletindo somente o instante em que foi medida a pressão arterial ${ }^{2}$.

A utilização da MAPA permite-nos estudar o padrão normal de pressão arterial ${ }^{3,4}$, as lesões em órgãos alvos decorrentes da hipertensão ${ }^{5}$, o prognóstico de eventos cardiovasculares e mortalidade ${ }^{6,7}$ e a análise da eficácia das drogas anti-hipertensivas ${ }^{8,9,10}$.
A MAPA é hoje um procedimento, efetivamente, incorporado à prática clínica.

Em 1987, Garret e Kaplan ${ }^{11}$ em editorial do Journal of Clinic Hypertension, colocavam-na como um procedimento promissor e cheio de ótimas perspectivas nas suas aplicações. Recentemente, $O$ Board of Trusttees of The American College of Cardiology aprovou parecer de uma comissão de renomados especialistas em hipertensão, sob o título "ACC Position Statement - Ambulatory Blood Pressure Monitoring ${ }^{12}$ que em resumo conclui: "A MAPA tornou-se um método maduro, clinicamente aplicável, com normatizações desenvolvidas por importantes sociedades e com consensos americanos e internacionais para suas indicações e procedimentos".

Por estas características do método e pelo momento atual pelo qual passa, justifica-se a abordagem deste importante assunto neste simpósio. 


\section{VANTAGENS, LIMITAÇÕES E PRINCIPAIS INDICAÇÕES}

As principais vantagens e as limitações da monitorização ambulatorial, em relação às medidas ca- suais da pressão arterial, são mostradas na Tabela I. Figuras 1 e 2.

Situações clínicas onde a MAPA tem utilidade e, portanto, indicação, estão expressas na Tabela II.

\section{Tabela I - Monitorização ambulatorial da pressão arterial: vantagens e limitações ${ }^{1}$}

\section{Vantagens:}

- Obtenção de múltiplas medidas

- Medidas durante as atividades usuais do paciente

- Ausência (ou redução) da reação de alerta

- Medidas durante o sono

- Ajuste dos intervalos de medidas

- Correlação com eventos ocorridos durante o exame

- Avaliação do ritmo circadiano da PA e freqüência cardíaca

- Possibilidade de análise de grande número de dados

\section{Limitações:}

- Perdas de dados (falhas técnicas)

- Distúrbios no trabalho e no sono

- Limitação da normatização de dados

- Falta de adequada avaliação dos equipamentos

- Alto custo

- Desconforto

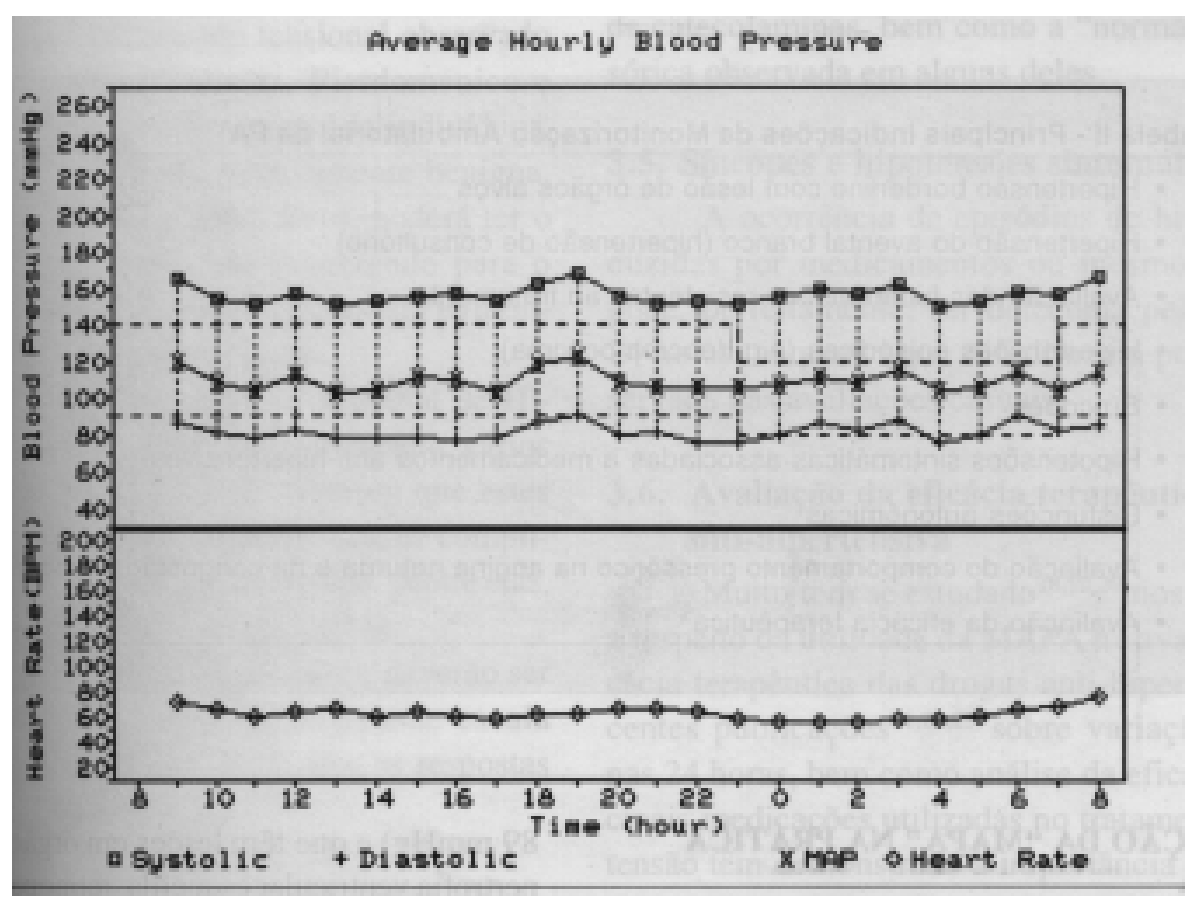

Figura 1. Monitorização Ambulatorial da Pressão Arterial de 24 horas, mostrando elevação sistemática da Pressão Arterial Sistólica. Observe-se um aspecto importante que a MAPA pode propiciar que é a análise do comportamento da pressão entre os períodos de vigília e sono, neste caso pode-se verificar ausência de queda da PA, entre os dois períodos analisados. Este fato, quando presente em pacientes hipertensos, relaciona-se, fortemente, com maiores probabilidades de complicações cardiovasculares, decorrentes da hipertensão e lesões em órgãos-alvos, podendo pois se constituir em um importante marcador prognóstico. 


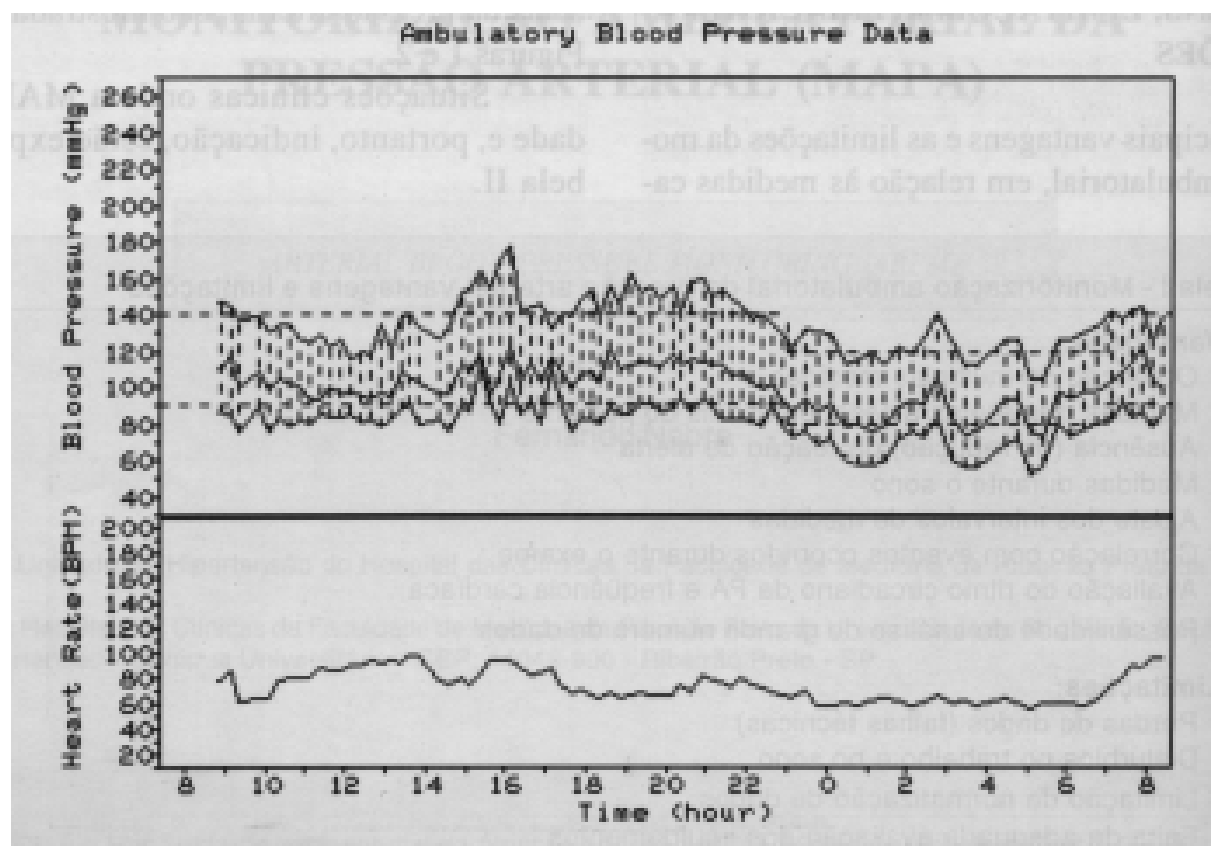

Figura 2. MAPA em paciente portadora de Feocromocitoma produtor de Noradrenalina. Observa-se presença de picos tensionais e alta variabilidade da pressão. A análise das médias tensionais entre os períodos de vigília e sono revelou descenso atenuado, fato este comum nos casos de hipertensão secundária.

\section{Tabela II - Principais indicações da Monitorização Ambulatorial da PA ${ }^{1}$}

- Hipertensão borderline com lesão de órgãos alvos

- Hipertensão do avental branco (hipertensão de consultório)

- Avaliação das hipertensões resistentes ao tratamento

- Hipertensões episódicas (e.g. feocromocitoma)

- Síncopes

- Hipotensões sintomáticas associadas a medicamentos anti-hipertensivos

- Disfunções autonômicas

- Avaliação do comportamento pressórico na angina noturna e na congestão pulmonar

- Avaliação da eficácia terapêutica

3. UTILIZAÇÃO DA “MAPA” NA PRÁTICA CLÍNICA

\subsection{Hipertensão "borderline" e presença de lesões em órgãos alvos}

Ocasionalmente, indivíduos que apresentam pressões do tipo "normal alto" (valores entre 85 e
$89 \mathrm{mmHg}$ ) e que têm lesões em órgãos alvos (e.g. hipertrofia ventricular esquerda, retinopatia, etc.) podem, quando submetidos à MAPA, mostrar níveis de PA, significativamente, mais elevados relacionados a estresse, esforço físico e/ou mental, tipo de trabalho, evidenciando que, nestes casos, a PA obtida no consultório foi subestimada. 


\subsection{Hipertensão do avental branco ou hiper- tensão do consultório}

Pickering e O'Brien ${ }^{13}$ afirmam, categoricamente, que a mais importante das aplicações clínicas da MAPA consiste na possibilidade de avaliar, adequadamente, os pacientes que apresentam um comportamento anormal da PA, quando examinados no consultório, mas não o reproduzem na observação de 24 horas. Kracoff e cols. ${ }^{14}$ estimam que o número de pacientes com hipertensão leve, em tratamento medicamentoso anti-hipertensivo, poderia ser reduzido em torno de $75 \%$, sem que a mortalidade aumentasse.

Nós, também, não temos dúvidas de que esta possibilidade atraente e prática de se excluir um contingente aproximado de 20 a $40 \%$ dos hipertensos leves e moderados, qualifique a MAPA como método dos mais úteis para este fim.

Alguns estudos sugerem ${ }^{15,16}$ que grande parte destes pacientes, que têm pressão arterial alterada no consultório, mas têm curvas e valores médios de pressão de 24 horas normais, não precisam ser tratados porque não reproduzem, em suas atividades habituais, o mesmo comportamento tensional observado frente ao médico. Recentemente, Pierdomenico e cols. ${ }^{17} \mathrm{de}-$ monstraram que este grupo de indivíduos, em função de uma evolução relativamente benigna, sem comprometimento de órgão alvos, poderá ter o seu tratamento medicamentoso postergado para o momento, se este ocorrer, desde que exista evidências de uma hipertensão estabelecida.

Durante o último Congresso Mundial de Hipertensão, realizado em Melbourne, Austrália, vários trabalhos, ainda não publicados, mostram que estes pacientes apresentam maior probabilidade de complicações decorrentes de hipertensão arterial, dentre elas, alterações mais freqüentes de órgão alvos.

Estes aspectos, ainda por resolver, deverão ser motivo de estudos prospectivos de grande escala em futuro próximo, para que tenhamos as respostas necessárias e esperadas.

\subsection{Hipertensão resistente à terapêutica}

Considera-se que a hipertensão é resistente ao tratamento quando não há resposta adequada pelas avaliações de consultório, em um paciente submetido a tríplice terapia. Entretanto, muitas vezes o efeito "Avental Branco" é o responsável por esta inadequa- da resposta hipotensora. Se há indícios de que a PA está controlada, por exemplo através de avaliações domiciliares, a MAPA está indicada para a análise da eficácia da medicação em uso. Há, também, de se considerar as características individuais de cada paciente, frente à ação dos medicamentos que estão sendo utilizados, fato no qual a monitorização da pressão arterial pode colaborar de maneira importante. Figuras 3 e 4.

\subsection{Hipertensões episódicas}

A situação mais freqüente que retrata episódios isolados de hipertensão é a ocorrência do feocromocitoma. Ainda, podemos observar picos hipertensivos em pacientes com síndrome de ansiedade ou de outras formas de desordens psíquicas. Em geral, como forma de diferenciação, não absoluta, mais possível, entre as duas situações está a ausência de descenso da pressão durante o sono, muito comum nas hipertensões secundárias. Temos, também, que lembrar a ausência de elevações ocasionais da PA em boa parte dos pacientes portadores deste tumor secretor de catecolaminas, bem como a "normalidade" pressórica observada em alguns deles.

\subsection{Síncopes e hipotensões sintomáticas}

A ocorrência de episódios de hipotensões induzidas por medicamentos ou mesmo de síncopes pode, perfeitamente, ser detectada pela MAPA em 24 horas, enquanto que dificilmente poderia ser observada nas avaliações casuais.

\subsection{Avaliação da eficácia terapêutica anti-hipertensiva}

Muito tem se estudado ${ }^{18,19,20}$ nos últimos anos a respeito da utilidade da MAPA na avaliação da eficácia terapêutica das drogas anti-hipertensivas. Recentes publicações ${ }^{21,22,23}$ sobre variação pressórica nas 24 horas, bem como análise da eficácia das principais medicações utilizadas no tratamento da hipertensão têm demonstrado a importância destes aspectos no prognóstico dos pacientes hipertensos. Nós mesmos utilizamos a monitorização ambulatorial da MAPA em algumas investigações ${ }^{24,25}$ com medicamentos anti-hipertensivos, objetivando a análise de sua eficácia terapêutica. Figuras 5, 6 e 7. 


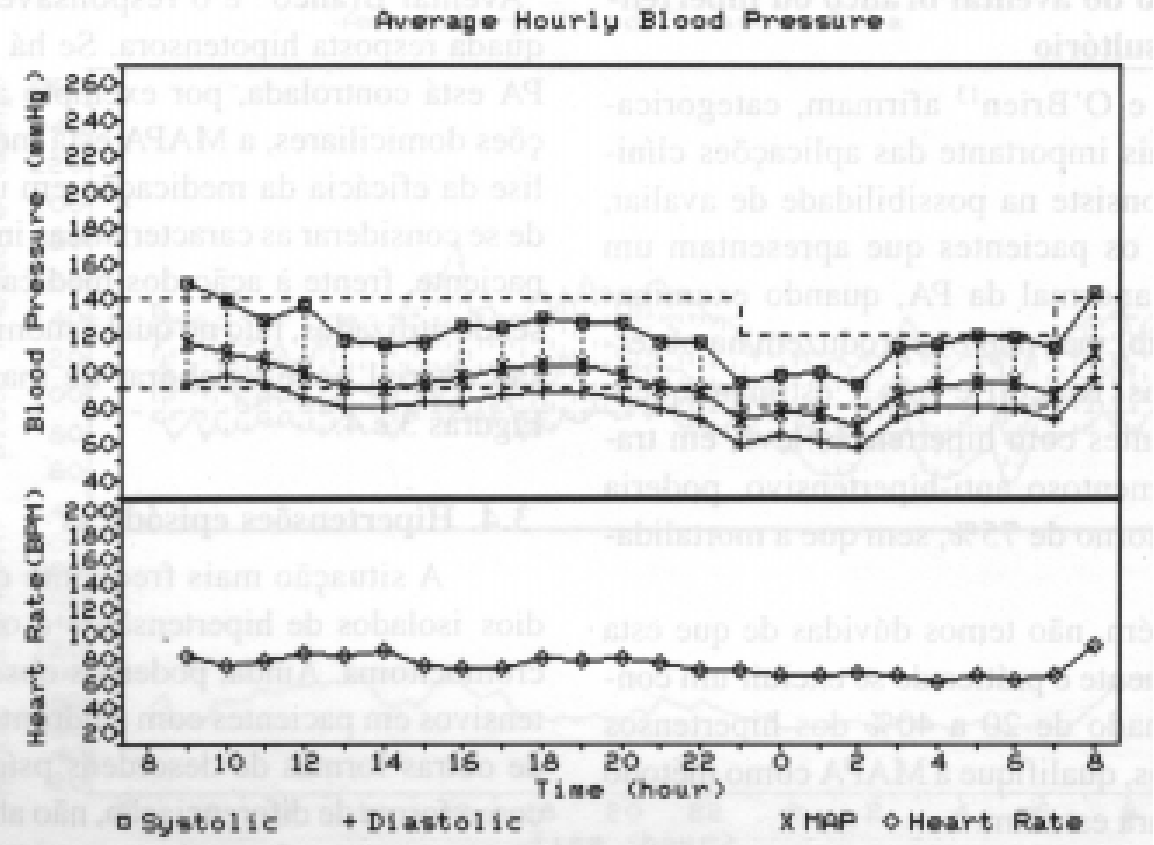

Figura 3. Paciente apresentando níveis tensionais, sistemáticamente, elevados em medidas casuais. Revelava, entretanto, medidas domiciliares normais. MAPA demonstrando elevação da pressão somente no início e términio do exame, (medidas que habitualmente são realizadas na clínica), com comportamento normal da pressão, nas 24 horas. Trata-se de um caso de "Hipertensão do Avental Branco".

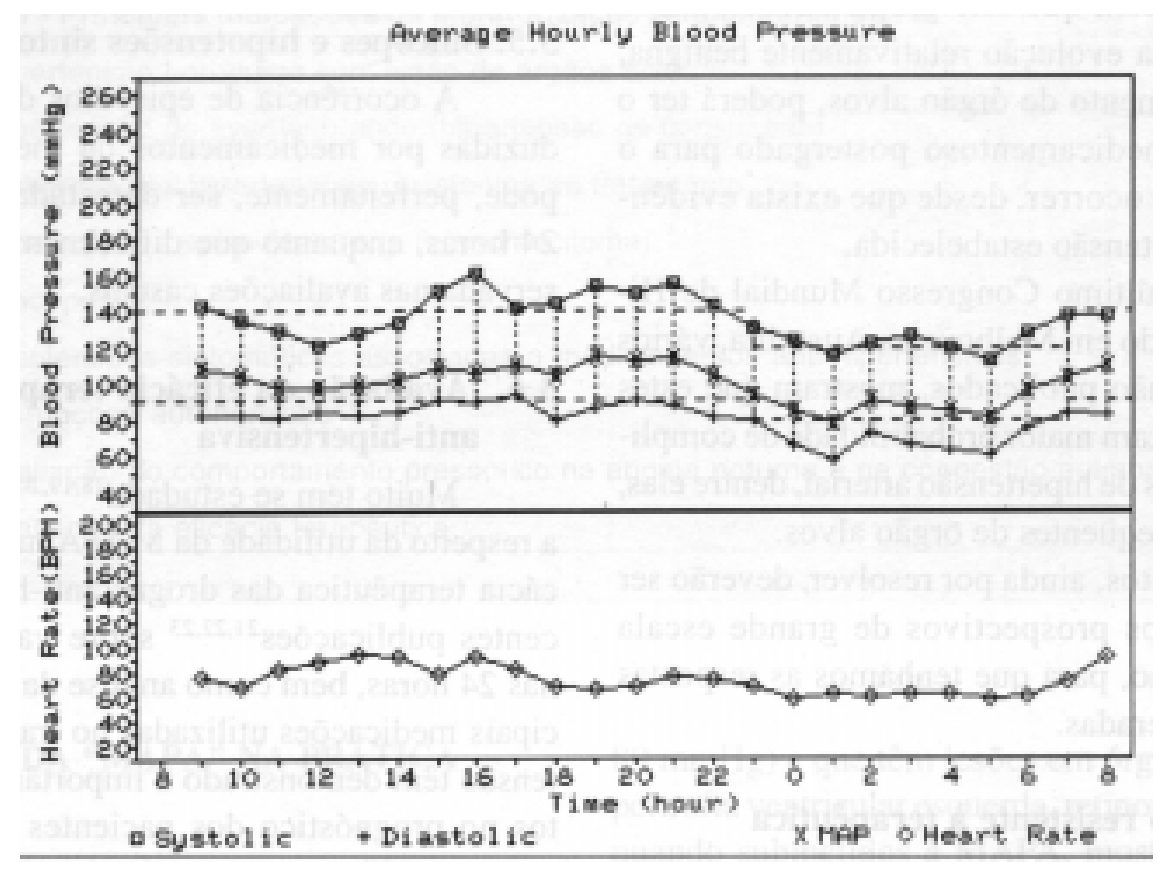

Figura 4. Exame realizado para avaliação da eficácia da terapêutica em uso. Pode-se observar que a paciente mantém PA controlada até por volta de 15 horas, quando há escape do controle tensional, permanecendo a pressão sistólica elevada até 22 horas. Nestas circunstâncias, a monitorização pôde indicar a necessidade de ajuste terapêutico para controle adequado e contínuo da pressão, nas 24 horas. 


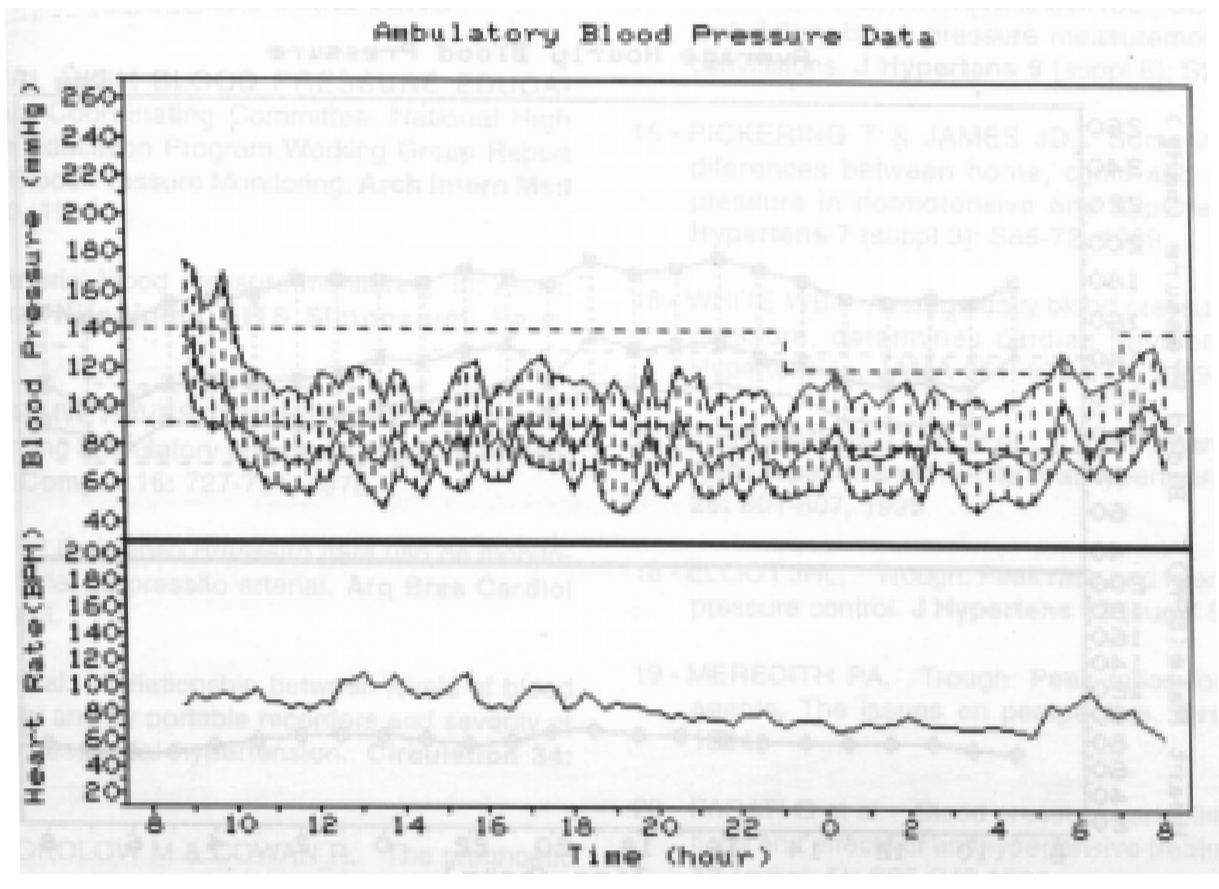

Figura 5. Neste caso, a situação clínica era semelhante à relatada na Figura 3. Observa-se, entretanto, a acentuada resposta hipertensiva que se observa no início do exame em contraposição a um comportamento tensional, praticamente, normal durante todo o restante do exame.

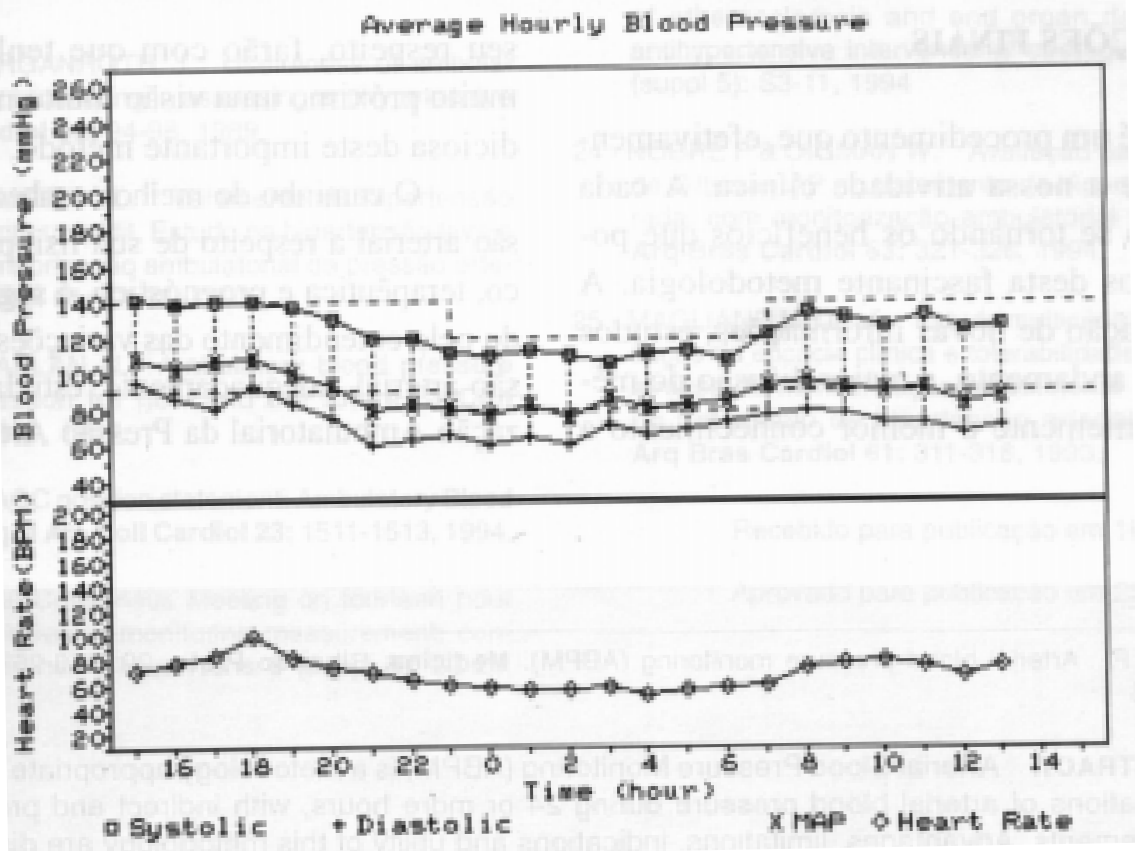

Figura 6. Paciente apresentando, durante as consultas médicas, níveis tensionais elevados a despeito do uso regular da medicação prescrita. A monitorização da pressão, em 24 horas, mostrou que a PA mantinha-se adequadamente controlada. Trata-se de um caso onde se observa o chamado "Efeito do Avental Branco". 


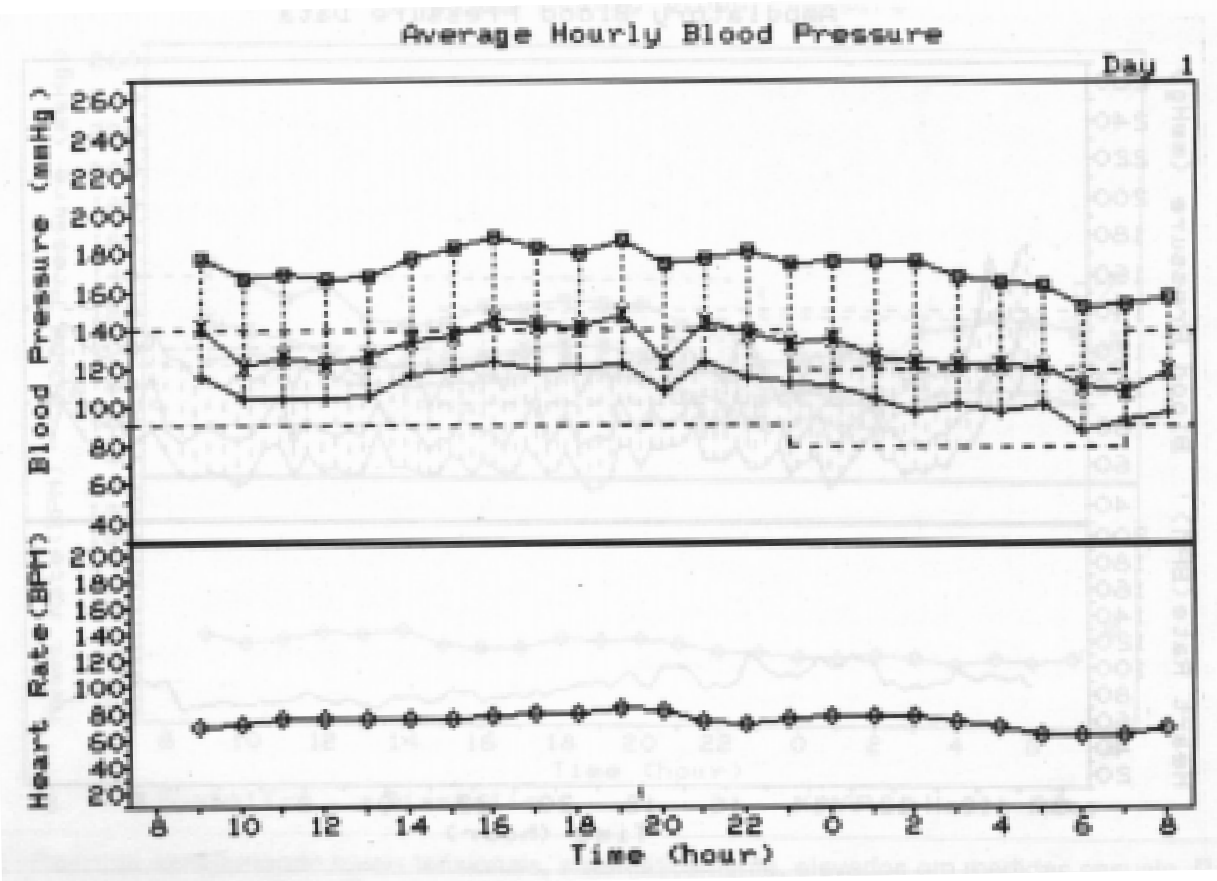

Figura 7. Exame realizado para avaliação de eficácia da terapêutica em uso. Observa-se, claramente, que o esquema posológico vigente não está sendo adequado para a manutenção da pressão nos níveis desejados. Pode-se, também, observar que não há o descenso fisiológico esperado da pressão entre a vigília e o sono.

\section{CONSIDERAÇÕES FINAIS}

A MAPA é um procedimento que, efetivamente, incorporou-se a nossa atividade clínica. A cada dia, maiores vão se tornando os benefícios que podem ser auferidos desta fascinante metodologia. A crescente publicação de novas informações, estudos prospectivos em andamento, a maior difusão do método e conseqüentemente o melhor conhecimento a seu respei- to, farão com que tenhamos num futuro muito próximo uma visão muito mais adequada e judiciosa deste importante método.

O caminho do melhor conhecimento da hipertensão arterial a respeito de sua fisiopatologia, diagnóstico, terapêutica e prognóstico, é seguramente constituído pelo entendimento das variações dinâmicas da pressão arterial, adequadamente, estudadas pela Monitorização Ambulatorial da Pressão Arterial de 24 horas.

NOBRE F. Arterial blood pressure monitoring (ABPM). Medicina, Ribeirão Preto, 29: 250-257, apr./sept. 1996

ABSTRACT: Arterial Blood Pressure Monitoring (ABPM) is a metodology appropriate to analyse the variations of arterial blood pressure during 24 or more hours, with indirect and programable measurements. Advantages, limitations, indications and utility of this metodology are discussed in this review.

UNITERMS: Hypertension. Blood Pressure Monitors. 


\section{REFERÊNCIAS BIBLIOGRÁFICAS}

1 - THE NATIONAL HIGH BLOOD PRESSURE EDUCATION. Program Coordinating Committee. National High Blood Pressure Education Program Working Group Report on Ambulatory Blood Pressure Monitoring. Arch Intern Med 150: 2270-2280, 1990.

2 - ELLIOT HL. Arterial blood pressure monitoring. In: Anals. II International Nifedipine GITS Simposium. Paris, p. 17-20, 1994

3 - MILLAR - CRAIG MW; HAVES, D \& WHITTINGTON J. New sistem for recording ambulatory and blood pressure in man. Med Biol Eng Comput 16: 727-731, 1978.

4 - NOBRE F et al. I Consenso Brasileiro para uso de monitorização ambulatorial da pressão arterial. Arq Bras Cardiol 60: 129-134, 1993.

5 - SOKOLOW M et al. Relationship between levels of blood pressure casually and by portable recorders and severity of complications in essential hypertension. Circulation 34: 279-298, 1966.

6 - PERLOFF B; SOKOLOW M \& COWAN R. The prognostic value of ambulatory blood pressure monitoring in treated hypertensive patients. J. Hypertens 9 (suppl 1): S33-S40, 1991.

7 - PICKERING TG \& DEVEREUX RB. Ambulatory monitoring of blood pressure as a predictor of cardiovascular risk. Am Heart J 114: 925-928, 1987.

8 - WHITE WB. Analysis of ambulatory blood pressure data in antihypertensive drug trials. J Hypertens 9 (suppl 1): S27-32, 1991.

9 - WHITE WB \& MORGANROTH J. Usefulness of ambulatory blood pressure monitoring in assessing antihypotensive therapy. Am J Cardiol 63: 94-98, 1989.

10 - NOBRE F \& NOGUEIRA JL. Tratamento da hipertensão arterial sistêmica com urapidil. Estudo na hipertensão leve e moderada com monitorização ambulatorial da pressão arterial. Arq Bras Cardiol 61: 63-68, 1993.

11 - GARRET NB \& KAPLAN N. Ambulatory blood pressure monitoring. A question for now and the future. J Clin Hipertens 3: 378-380, 1987

12 - SHEPS SG et al. ACC position statement: Ambulatory Blood Pressure Monitoring. J Am Coll Cardiol 23: 1511-1513, 1994.

13 - Second International Consensus Meeting on fourteen hour blood pressure ambulatory monitoring measurement: consensus and conclusions. J Hypertens 9 (suppl 8): S2-26, 1991.
14 - SECOND INTERNATIONAL CONSENSUS. Meeting on 24 ambulatory blood pressure measurement: consensus and convlusions. J Hypertens 9 (suppl 8): S28-S30,1991.

15 - PICKERING T \& JAMES JD. Some implications of the diferences between home, clinic and ambulatory blood pressure in normotensive and hypertensive patients. $J$ Hypertens 7 (suppl 3): S65-72, 1989.

16 - WHITE WB. Average daily blood pressure, not office blood pressure, determines cardiac function in patients with $\mathrm{Hy}$ pertension. JAMA 261: 873-877, 1989.

17 - PIERDOMENICO SD et al. Target organ status and serum lipids in patients with white coat hypertension. Hypertension 26: 801-807, 1995.

18 - ELLIOT JHL. Trough: Peak ratio and twenty-four-hour blood pressure control. J Hypertens 12 (suppl 5): S29-S33, 1994.

19 - MEREDITH PA. Trough: Peak ratios for antihypertensive agents. The issues en perspective. Drugs 48: 661-666, 1994.

20 - PARATI G et al. Blood pressure variability: clinical implications and effects of antihypertensive treatment. J Hypertens 12 (suppl. 5): S35-S40,1994.

21 - PARATI G et al. Blood pressure variabillity: clinical implications and effects of antihypertensive treatment. $\mathbf{J}$ Hypertens 12 (suppl 5): S35-40, 1994

22 - COCA A. Circadian rhythm and blood pressure control: physiological and pathophysiological factors. J Hypertens 12 (suppl 5): S13-21, 1994.

23 - COLIN JS; VALENTE AJ \& HILDEBRANDT EF. Prevention of atherosclerosis and end organ damage: a basis for antihypertensive interventional strategies. J Hypertens 12 (suppl 5): S3-11, 1994

24 - NOBRE F \& OIGMAN W. Avaliação da eficácia terapêutica de Diltiazen AP no tratamento da hipertensão leve à moderada, com monitorização ambulatorial da pressão arterial. Arq Bras Cardiol 63: 321-326, 1994.

25 - MAGLIANO MF et al. Estudo multicêntrico brasileiro de avaliação da eficácia clínica e tolerabilidade da isradipina SRO, através de monitorização ambulatorial da pressão arterial, no tratamento da hipertensão arterial leve e moderada. Arq Bras Cardiol 61: 311-318, 1993.

Recebido para publicação em 16/01/96

Aprovado para publicação em 25/04/96 